\title{
Primer registro de Heliotropium amplexicaule (Heliotropiaceae) en Chile
}

\section{First record of Heliotropium amplexicaule (Heliotropiaceae) in Chile}

\author{
Sergio Ibáñez ${ }^{1}$, Federico Luebert ${ }^{2,3}$ \& Miguel Gómez ${ }^{1}$ \\ ${ }^{1}$ Departamento de Ciencias Vegetales, Facultad de Agronomía e Ing. Forestal, Universidad Católica de Chile. Avenida Vicuña \\ Mackenna 4860, Santiago, Chile. \\ ${ }^{2}$ Freie Universität Berlin, Institut für Biologie-Botanik, Berlín, Alemania. \\ ${ }^{3}$ Departamento de Silvicultura, Facultad de Ciencias Forestales y Conservación de la Naturaleza, Universidad de Chile, \\ Santiago, Chile. \\ stibanez@uc.cl, f.luebert@fu-berlin.de, mgomezu@uc.cl
}

\begin{abstract}
The presence of the commonly known weed Heliotropium amplexicaule (Heliotropiaceae) is reported for Chile first time, from a collection recently made in Santiago. Its potential as weed in Chile is discussed in the context of the experience of other countries where the species has become naturalised.
\end{abstract}

El género Heliotropium L. (Heliotropiaceae) en su circunscripción actual comprende cerca de 300 especies distribuidas principalmente en las regiones tropicales y subtropicales de todos los continentes (Johnston 1928, Förther 1998, Hilger \& Diane 2003, Luebert et al. en prensa). En Chile, el género Heliotropium está representado por 24 especies nativas (Zuloaga et al. 2008), de las que 17 pertenecen a la sección Cochranea (Luebert 2010). En cuanto a las especies introducidas, sólo dos se han reportado en Chile: $H$. angiospermum Murray y $H$. procumbens Mill. (Marticorena \& Quezada 1985). La primera es una especie que se distribuye desde el sur de los Estados Unidos hasta el sur de Perú, mientras que la segunda está ampliamente distribuida en Sudamérica tropical, el Caribe, América central y sur de los Estados Unidos (Johnston 1928, 1964, Macbride 1960, Melo \& Semir 2008). De acuerdo con Arroyo et al. (2000) Heliotropium angiospermum se ha naturalizado en la Región de Arica y Parinacota. Sin embargo, la presencia de $H$. procumbens en Chile es dudosa: El único material sobre la base del que se ha citado Heliotropium procumbens para Chile es el tipo de $H$. simplex Meyen (tipo B, destruido), que Johnston (1928) trata como un sinónimo de H. procumbens. Sin embargo, Johnston (1937: 17), después de revisar el material en $\mathrm{B}$, concluye que $H$. simplex es un sinónimo de H. angiospermum.

Heliotropium amplexicaule Vahl es una especie nativa del norte y centro de Argentina, sur de Bolivia, Uruguay y el extremo sur de Brasil (Johnston 1928). Pertenece a la sección Heliotrophytum, en que la totalidad de las especies son de origen sudamericano (Johnston 1928, Förther 1998). Esta especie ha sido introducida en África (Martins 1990), Europa (Brummitt 1972), Norteamérica (Johnston 1964, Munz \& Keck 1965) y Oceanía (Craven 1996). Sin embargo, su presencia en Chile sólo había sido referida por F. Philippi (1881: 211), bajo el sinónimo Heliotropium anchusaefolium Poir., sobre la base de lo que dijo R.A. Philippi (1870: 190), que lo señala para Mendoza. En este trabajo se reporta por primera vez en Chile la presencia de $H$. amplexicaule, basado en material recolectado en la ciudad de Santiago.

Heliotropium amplexicaule Vahl., Symb. Bot. 3: 21, 1794. TIPO: Montevideo, P. Commerson s.n. (holotipo, C!; isotipo, P no visto [fototipo!]).

Sin.: Heliotropium anchusaefolium Poir., Encycl. 3: 23. 1803. H. bolivianum Rusby, Mem. Torrey Bot. Club 4: 225. 1895. H. lithospermifolium (DC.) Speg., Contr. Fl. Sierra Vent.: 44. 1896. Para una sinonimia detallada véase Johnston (1928, 1964), Gangui (1955), Förther (1998), Zuloaga et al. (2008).

Icon.: Gangui (1955: 529, fig. 9), Dawson (1965: 108, fig. 38), Pérez-Moreau \& Cabrera (1983: 266, fig. 107A-E), Pérez-Moreau (1994: 19, fig. 7a-e), Melo \& Semir (2008: 758, figs. 1-6).

Hierba perenne a subarbusto, de tallos decumbentes o erectos. Es muy variable en hábito, forma de la hoja y 
color de la corola, con hojas sésiles, linear-lanceoladas a lanceolado-oblongas y corolas blancas a violeta (Fig. 1). El estigma es sésil y el fruto se divide en dos nueces biseminadas, bicarpelares y puede formar cavidades estériles. Se diferencia de Heliotropium nicotianifolium Poir. y H. leiocarpum Morong, también pertenecientes a la sección Heliotrophytum (Johnston 1928, Förther 1998) por poseer hojas caulinares sésiles, respecto de hojas pecioladas en las dos últimas especies. La otra especie de la sección Heliotrophytum, $H$. phylicoides, presenta hojas sésiles, pero se diferencia de $H$. amplexicaule en que en esta última las hojas y los tallos poseen pubescencia glandulosa caférojiza, mientras que en la primera la pubescencia es blancovillosa y sin glándulas aparentes. Johnston (1928: 21) proporciona una clave de identificación para las especies de esta sección.
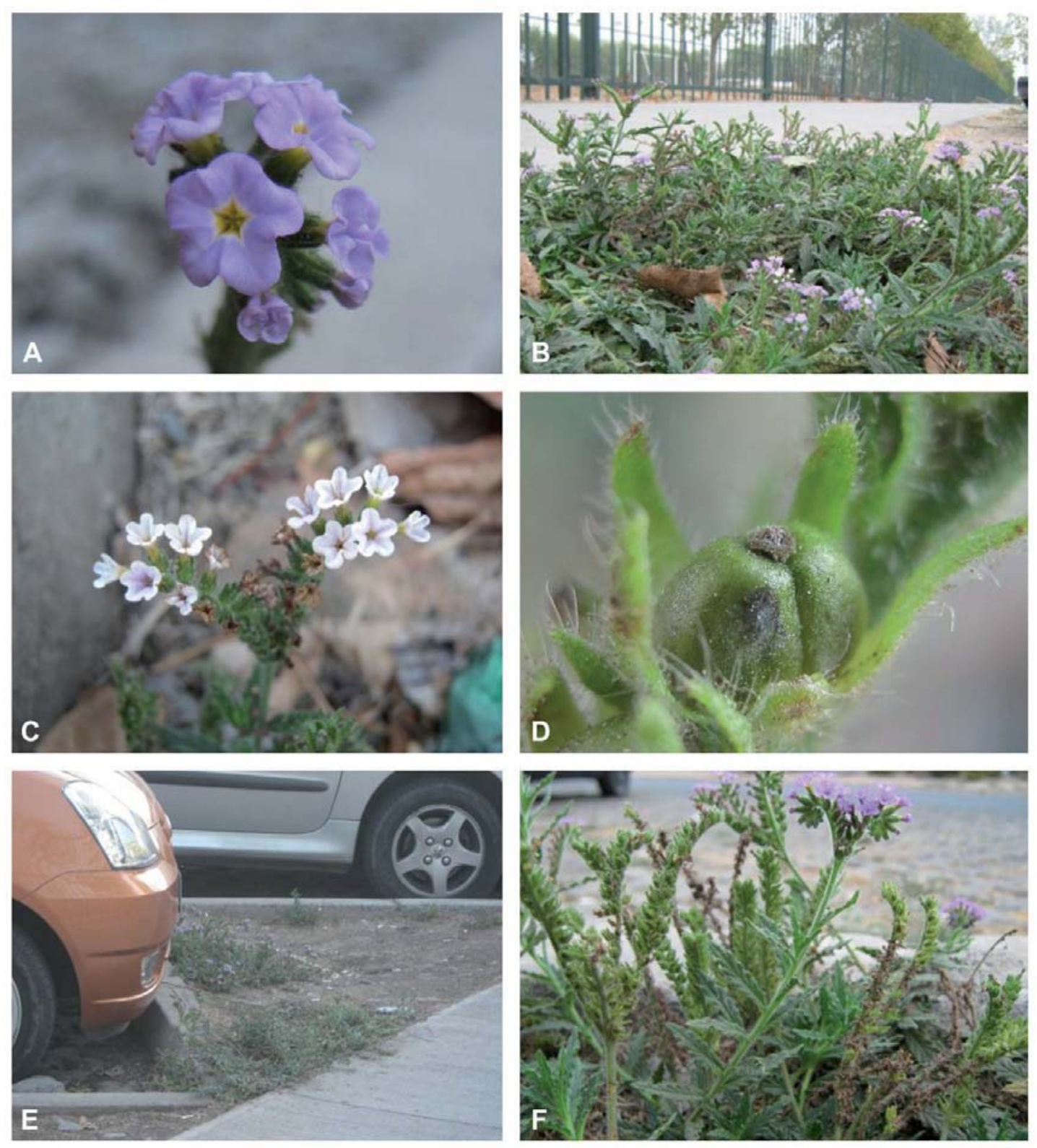

Figura 1. Heliotropium amplexicaule creciendo en Chile A. Flores. B. Hábito de la especie. C. Inflorescencia de flores blancas. D. Fruto. E. Individuos creciendo en la calle. F. Rama con inflorescencia (Fotografías S. Ibáñez).

Figure 1. Heliotropium amplexicaule growing in Chile A. Flowers. B. Habit of the species. C. White flowers inflorescence. D. Fruit. E. Individuals growing in the street. F. Branch with inflorescence (Photograps S. Ibáñez). 
Presencia en Chile

Heliotropium amplexicaule sólo se ha encontrado en Chile en la comuna de Macul, ciudad de Santiago (Fig. 1). La población detectada crece en sitios eriazos próximos al campus San Joaquín de la Universidad Católica de Chile, junto a Convolvulus arvensis L., Polygonum aviculare L. y Euphorbia serpens Kunth. Al parecer se encuentra actualmente en condición de alóctona o casual (sensu Richardson et al. 2000). No se detectó su presencia en otros lugares adyacentes a más de $100 \mathrm{~m}$ de distancia y no hay señales de que la especie se haya naturalizado.

La morfología de las plantas encontradas coincide con la descripciones y materiales de herbario examinados de Heliotropium amplexicaule, ya que presenta hojas rugosas, glandulosas, sésiles, oblongo-lanceoladas de margen sinuado, y flores con estigma sésil.

Material estudiado: CHILE, Región Metropolitana, Prov. Santiago, Campus San Joaquín, Macul, 33³0’05”S; 70³6’30’’W, 577 m s.n.m., 6-IV-2010, S. Ibáñez 01 (SGO).

Material adicional estudiado (selección): ARGENTINA, Prov. Buenos Aires, Pigue, 13-XII-1938, W.J. Eyerdam, A.A. Beetle \& E. Grondona 23401 (G); Prov. Córdoba, Deán Funes, 2-XI-1945, A.R. Cuezzo 730 (BM); Prov. Corrientes, ruta 23, 20 km E de Mercedes, 29-X-1973, A. Schinini 7571 (G); Prov. Entre Ríos, Salto Grande, Río Uruguay, Casa de Piedra, 15 m, 30-X-1978, S.A. Renvoize 2868 (B); Prov. Salta, Alrededores del pueblo de Amblayo, 2.300-2.600 m, 1-IV-1990, L. Novara \& R. Neuman 715 (G); Prov. Tucumán, Tafí del Valle, al pie del Cerro Pelado, 2.200 m, 24-I-1950, H. Sleumer 129 (B). BOLIVIA, Depto. Cochabamba, Vic. Cochabamba, 1891, M. Bang 926 (BM, G, GH, isotipos de H. bolivianum). Depto Chuquisaca, La Compuerta, carretera de Sucre a Chiqui Chiqui, 1850.5'S, 65².8’W, 2.356 m, 5-VIII-2007, M. Velayos, C. Aedo \& C. Monge 11180 (MA). BRASIL, Brasilia meridionalis, F. Sellow s.n. (G-DC, holotipo de $H$. lithospermifolium). URUGUAY, Depto. Montevideo, Atahualpa, XI-1925, W.G.F. Herter 79759 (F).

\section{IMPORTANCIA COMO MALEZA}

Tanto en su distribución original como en los lugares donde es alóctona, Heliotropium amplexicaule es considerada una planta dañina, debido a su carácter invasor por un rizoma que permite la reproducción asexual (Briese et al. 2000), facilitada por las faenas de labores del suelo. Las semillas germinan principalmente entre finales del verano e inicios de otoño (Parsons \& Cuthbertson 2001). Esta especie tiene la capacidad de establecerse en una amplia gama de suelos (Craven 1996) y es común invadiendo praderas de pastoreo, donde puede cubrir grandes parches, afectando la productividad (Parsons \& Cuthbertson 2001). También es consumida por los animales, pudiendo causar toxicidad e incluso la muerte, debido a una pirrolizidina llamada indicina (Glover \& Ketterer 1987). Por su carácter invasor, su presencia en Chile podría constituir un riesgo, que debe ser confirmado con futuros hallazgos.

\section{AGRADECIMIENTOS}

Los autores agradecen a Mélica Muñoz y Gloria Rojas por su atención y buena disposición en el herbario del Museo de Historia Natural, y a Jorge Macaya por el material bibliográfico aportado. A los curadores de los herbarios $\mathrm{B}$, BM, C, F, G, GH, MA, SGO por facilitar acceso a material de herbario citado en este trabajo. F. Luebert agradece el apoyo financiero del Servicio Alemán de Intercambio Académico, DAAD. Esta investigación recibió apoyo del proyecto SYNTHESYS http://www.synthesys.info/ que es financiado por la European Community Research Infrastructure Action bajo los programas FP6 "Structuring the European Research Area” y FP7 “Capacities”.

\section{BIBLIOGRAFÍA}

Arroyo, M.T.K., C. Marticorena, O. Matthei \& L. Cavieres. 2000. Plant invasions in Chile: Present patterns and future predictions. In: H.A. Mooney \& R.J. Hobbs (eds.), Invasive species in a changing World, pp. 385-421. Island Press, New York.

Briese, D.T., D.A. Mclaren, W.J. Pettit, M. Zapater, F. Anderson, R. Delhey \& R. Distel. 2000. New biological control initiatives against weeds of South American origin in Australia: Nassella tussock grasses and blue heliotrope. In: N.R. Spencer (ed.), Proceedings of the X International Symposium on Biological Control of Weeds, pp. 215-223. Montana State University, Bozeman, Montana.

BrummitT, R.K. 1972. Heliotropium L. In: T.G. Tutin, V.H. Heywood, N.A. Burges, D.M. Moore, D.H. Valentine, S.M. Walters \& D.S. Webb (eds.), Flora Europaea, Vol. 3, pp. 84-86. Cambridge University Press, Cambridge.

Craven, L.A. 1996. A taxonomic revision of Heliotropium (Boraginaceae) in Australia. Australian Systematic Botany 9(4): 521-657.

Dawson, G. 1965. Boraginaceae. En: A.L. Cabrera (ed.), Flora de la Provincia de Buenos Aires, pp. 100-121. Colección Científica del INTA, Buenos Aires.

Förther, H. 1998. Die infragenerische Gliederung der Gattung Heliotropium L. und ihre Stellung innerhalb der Subfam. Heliotropioideae (Schrad.) Arn. (Boraginaceae). Sendtnera 5: 35-241.

Gangui, N. 1955. Las especies silvestres de Heliotropium de la República Argentina. Revista de la Facultad de Ciencias Exactas, Físicas y Naturales 17: 481-560.

Glover, P.E. \& P.J. Ketterer. 1987. Blue heliotrope kills cattle. Queensland Agricultural Journal 113: 109-110.

Hilger, H.H. \& N. Diane. 2003. A systematic analysis of 
Gayana Bot. 68(1), 2011

Heliotropiaceae (Boraginales) based on trnL and ITS1 sequence data. Botanische Jahrbücher für Systematik, Pflanzengeschichte und Pflanzengeographie 125: 19-51.

Johnston, I.M. 1928. Studies in the Boraginaceae VII. The South American species of Heliotropium. Contributions from the Gray Herbarium of Harvard University 81: 3-73.

Johnston, I.M. 1937. Studies in the Boraginaceae XII. 2. Novelties and critical notes. Journal of the Arnold Arboretum 18: 1025.

Johnston, I.M. 1964. Boraginaceae. In: C.L. Lundell (ed.), Flora of Texas, Vol. 1, pp. 123-221. Texas Research Foundation, Renner.

Luebert, F. 2010. Systematics, ecology and evolution of Heliotropium sect. Cochranea and the biogeography of the Atacama Desert. Dissertation, Freie Universität Berlin, Berlin.

Luebert, F., G. Brokamp, J. Wen, M. Weigend \& H.H. Hilger. Phylogenetic relationships and morphological diversity in Neotropical Heliotropium (Heliotropiaceae). Taxon (en prensa).

Macbride, J.F. 1960. Flora of Peru: Boraginaceae. Field Museum of Natural History, Botanical Series 13(5, 2): 539-609.

Marticorena, C. \& M. Quezada. 1985. Catálogo de la flora vascular de Chile. Gayana Botánica 42(1-2): 1-157.

Martins, E.S. 1990. 116. Boraginaceae. In: E. Launert \& G.V. Pope (eds.), Flora Zambesiaca, vol. 7(4), pp. 59-114. Flora Zambesiaca Managing Committee, London.

Melo, J.I.M. \& J. Semir. 2008. Taxonomia do gênero Heliotropium
L. (Heliotropiaceae) no Brasil. Acta Botanica Brasilica 22: 754-770

Munz, P. \& D. Keск. 1965. A California flora. University of California Press, Berkeley. 1681 pp.

Parsons, W.T. \& E.G. Cuthbertson. 2001. Noxious weeds of Australia. CSIRO, Collingwood. 698 pp.

Pérez-Moreau, R.L. 1994. Boraginaceae. En: R.L. Pérez-Moreau (ed.), Flora Chaqueña, Vol. 8, pp. 3-35. Instituto Nacional de Tecnología Agropecuaria, Buenos Aires.

Pérez-Moreau, R.L. \& A.L. Cabrera. 1983. Boraginaceae. En: A.L. Cabrera (ed.), Flora de la provincia de Jujuy, República Argentina, Vol. 8, pp. 247-291. Colección Científica del INTA, Buenos Aires.

Philippi, F. 1881. Catalogus plantarum vascularium chilensium adhuc descriptarum. Imprenta Nacional, Santiago, Chile. 378 pp.

PhilipPI, R.A. 1870. Sertum mendocinum alterum, o sea, catálogo de las plantas recojidas cerca de Mendoza y en los caminos que conducen de Chile a esa ciudad. Anales Universidad de Chile 36: 159-212.

Richardson, D.M., P. Pyšek, M. Rejmánek, M.G. Barbour, F.D. Panetta \& C.J. West. 2000. Naturalization and invasion of alien plants: Concepts and definitions. Diversity and Distributions 6(2): 93-107.

Zuloaga, F.O., O. Morrone \& M.J. Belgrano (eds.). 2008. Catálogo de las plantas vasculares del Cono Sur (Argentina, Sur de Brasil, Chile, Paraguay y Uruguay), Vol. 2. Missouri Botanical Garden Press, St. Louis, 985-2286.

Recibido: 16.11 .10

Aceptado: 05.01.11 